\title{
10 top tips for managing the older dentition
}

\section{By Dr Antony J. Preston'}

\section{Introduction}

The oro-dental management of the older patient can be challenging. There are a variety of social, medical and oro-dental issues affecting the more mature patient in the present day that, arguably, can make their dental treatment more difficult than that of several decades ago. In this paper, ten general tips will be described that might be of help for dental professionals when managing elderly patients.

\section{Be realistic when managing the elderly patient Keep it simple}

Perhaps the principle of 'Keep it simple' should not be exclusively attributed to the dental management of the elderly patient but for all patients. It is, however, especially true for the more elderly patient. Most of our prevention regimes and dental treatment modalities rely for their long-term success, on the compliance and co-operation of the patient. Oral hygiene and prosthesis hygiene is essential for the maintenance of a healthy mouth. If, however, one advises a more elderly patient to exercise the intricate use of dental floss to clean interdentally or the use of a single-tufted brush to clean the overly-complicated components of a 'busy' partial denture design, then this could be seen as unrealistic - especially if the patient suffers from conditions such as rheumatoid arthritis of the hands. The author is not saying that elderly patients should not clean their

Senior Lecturer/Honorary Consultant in Restorative Dentistry, School of Dentistry, University of Liverpool mouths or dentures, but rather that when advising a patient to implement such hygiene procedures, the methods advised should be realistic and 'do-able'. Partial denture designs should be kept as simple as at all possible in order to 'do the job' and intricate ('busy') partial denture designs with a plethora of minor components just serve as dental plaque traps, rather than doing the patient a service.

For the majority of elderly patients, sophisticated restorative dentistry and fixed prosthodontics work is probably not advisable - especially if access to dental care is an issue (if the patient is resident in a residential or nursing home) or if the patient is subject to dementia. For these types of patients, simple extractions, direct restorations and removable prostheses of relatively simple design are 'the way forward'. That is not to say that one should never implement fixed prosthodontic work for an elderly patient - but rather that case selection for this cohort of patients for this type of treatment is crucial.

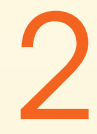

\section{Be aware of the potential of} drug-induced xerostomia The most common cause of xerostomia and difficult to manage

Approximately a decade ago, if I was writing this article, I would say that there were approximately 300 medications can have the potential side effect of causing drug-induced xerostomia (Fig. 1). This figure is now over 500 ! Many of our elderly patients are taking medications such as antihypertensives or antidepressants that can have this side effect.

Whether a patient is dentate, partially dentate or edentulous, being xerostomic can have a significant impact in terms of dental caries, root surface caries, toothwear, denture retention problems and oral ulceration and soreness. One must not underestimate the effect of drug-induced xerostomia and warn

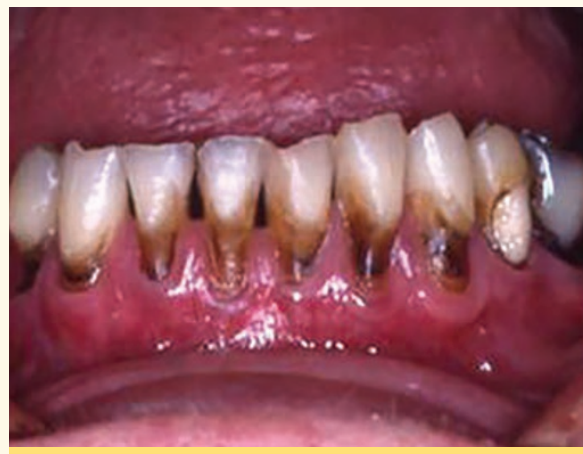

Fig. 1 Drug-induced xerostomia

the patient of its significance. The xerostomic (if dentate) is by definition at high risk for dental caries and so vigorous anti-caries prevention regimes should be instituted.

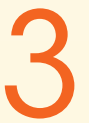

Be aware of the cariogenic potential of many elderly individuals' diets

Elderly individuals often have a highsugar diet and exposed root surfaces

It is true to say that many elderly individuals have a relatively high sugar, cariogenic diet.

Transport problems might present if the patient has to do their own shopping and so they might elect to carry lighter foodstuffs (such as confectionery) home from the supermarket, rather than heavier foodstuffs, such as fruit and vegetables.

The elderly patient might be widowed, depressed and living on their own, and so they might 'comfort eat' their cariogenic diet. This situation, from a dental caries point of view, will be compounded if the patient is taking an antidepressant medication that could cause drug-induced xerostomia. If exposed root surfaces are present in the patient's mouth, then root surface caries can progress at an alarming rate in such conditions. 


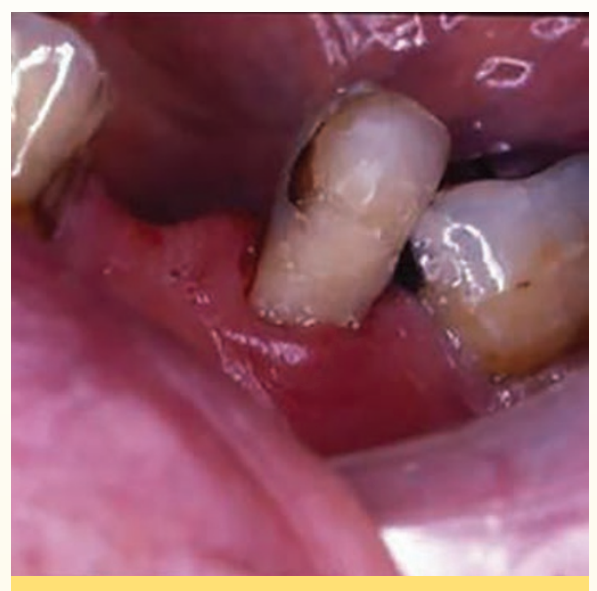

Fig. 2 Root surface caries restoration

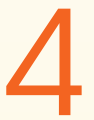

Consider the use of resinmodified glass ionomer

cements to restore root surface caries lesions

RMGICs are good material for root surface caries restoration

The main way that restorations fail in the restoration of root surface caries is through secondary dental caries at the restoration margin (Fig. 2). The fluoride release, and more-importantly, so-called fluoride 'recharge' capability of resin-modified glass ionomer cement (RMGIC) restorations can be a theoretical advantage in the prevention of such restorations' marginal secondary caries. The in vivo evidence, however, for this claim is not conclusive, at present.

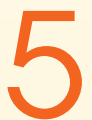

Consider the repair, rather than the replacement of defective (large) restorations Keep it simple

Again, the principle of 'keep it simple' applies to the situation whereby a large restoration in an elderly patient has fractured (Fig. 3). There is contemporaneous evidence to suggest that, in certain cases, the repair of such a restoration, rather than its complete replacement, is the preferable option (as long as any secondary dental caries is addressed).

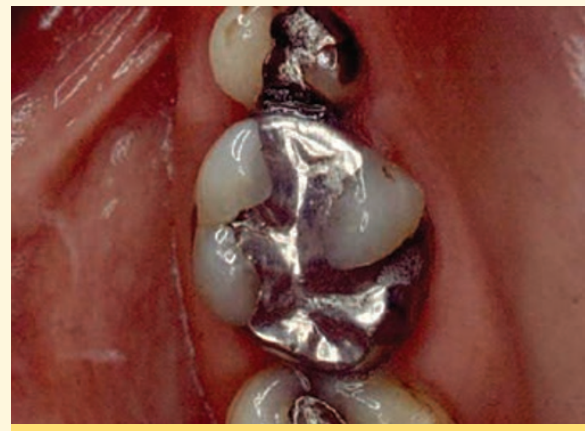

Fig. 3 Large restoration
6 Consider the use of the shortened dental arch concept in the mandible

. but might the use of the shortened dental arch mean more anterior toothwear?

The concept of the shortened dental arch (SDA) is well known to dental clinicians. Its use - especially in the mandible - is welldocumented. Experience shows us that the tolerance of many lower bilateral free-end or distal-extension saddle partial dentures can be poor and so in such cases, the acceptance of a lower SDA might be the preferred option. Some authorities, however, have expressed concern that such an approach might increase the risk of lower anterior toothwear and so this should be monitored.

\section{Consider the use of overdentures \\ An under-used but relatively 'simple' treatment modality}

Overdentures were a relatively popular treatment modality a few decades ago, but in recent years, they have fallen away a little in their popularity, perhaps due to the rise of other treatment modalities, such as dental implants. Overdentures, onlay dentures and overlay dentures still have a role to play in the management of many elderly patients as a relatively 'cheap and cheerful' way to manage their prosthodontic requirements.

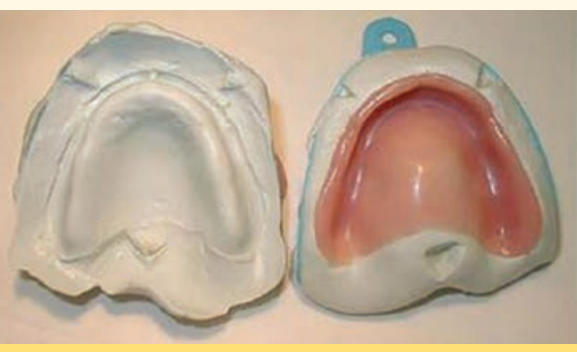

Fig. 4 Copy complete dentures

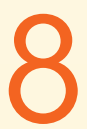

Consider the use of 'copy' complete dentures Habituation issues with age

As edentulous patients become older, their ability to accommodate to or habituate to subtle changes in the design of their complete dentures can decrease. In these situations, as long as it has been diagnosed that the tooth position or polished surface is acceptable, then the 'copy' denture or 'duplicate' denture treatment modality comes into its own (Fig. 4). If an elderly patient has many sets of dentures made in a relatively short number of years, each with subtle design differences, it often can be useful to ask the patient which set of dentures was the most comfortable or successful and then to use these dentures as the template for a copy denture technique.

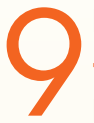

Be systematic at each stage - especially the treatment planning stage of complete denture fabrication

Be methodical when making C/C minimising errors

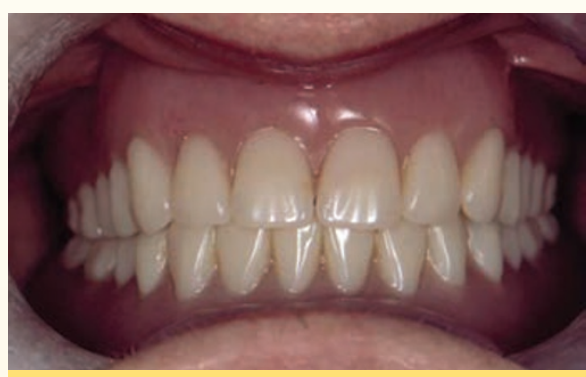

Fig. 5 Treatment planning for complete dentures

I often say to undergraduate dental students that the most important or 'clever' stage in complete denture fabrication is the treatment planning stage (Fig. 5). At the treatment planning stage, one should aim to make a 'shopping list' of those features of the existing complete dentures (if there are any) that are acceptable and should be mimicked in the replacement dentures, and those features that should be corrected. Only in this way, through the careful inspection of the occlusal, polished and fitting surfaces extra-orally and the fit, extensions, retention, stability, neutral zone, occlusion, articulation and aesthetics intra-orally, can one make a detailed diagnosis and plan of the patient's complete denture situation. Importantly, one should also make a realistic prognosis of the situation and tell the patient (perhaps repeated at each denture fabrication stage) $\ldots$ and record it in the patient's record notes.

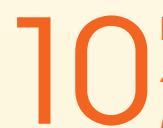

If in doubt - refer A second opinion or 'fresh pair of eyes' can often

be useful

A dental professional may want to be caring and compassionate in their desire to help elderly patients with their dental treatment requirements - but for the more challenging cases, it might be a good idea to have a second opinion to confirm one's treatment planning thoughts. That is not to say that every patient of extended years should be referred, but certainly, if a dental clinician has significant doubts as to the way in which to manage the more 'tricky' case, then a 'fresh pair of eyes' on the matter can often be valuable.

bdjteam2016172 